\title{
Holographic Abrikosov lattice: Vortex matter from black hole
}

\author{
Chuan-Yin Xia, ${ }^{1, *}$ Hua-Bi Zeng, ${ }^{1, \dagger}$ Yu Tian, ${ }^{2,3, \star}$ Chiang-Mei Chen $\odot,{ }^{4, \S}$ and Jan Zaanen $\oplus^{5, \|}$ \\ ${ }^{1}$ Center for Gravitation and Cosmology, College of Physical Science and Technology, \\ Yangzhou University, Yangzhou 225009, China \\ ${ }^{2}$ School of Physics, University of Chinese Academy of Sciences, Beijing 100049, China \\ ${ }^{3}$ Institute of Theoretical Physics, Chinese Academy of Sciences, Beijing 100190, China \\ ${ }^{4}$ Department of Physics, Center for High Energy and High Field Physics (CHiP), \\ National Central University, Chungli 32001, Taiwan \\ ${ }^{5}$ Institute Lorentz, for Theoretical Physics, Leiden University, P.O. Box 9506, \\ 2300 RA Leiden, The Netherlands
}

(Received 30 November 2021; accepted 20 December 2021; published 4 January 2022)

\begin{abstract}
The AdS/CFT correspondence provides a unique way to study the vortex matter phases in superconductors. We solve the dynamical evolution of a superconductor in $2+1$ dimensions at a finite temperature subjected to a magnetic field quench in terms of a gravitational "hairy black hole" dual living in an asymptotic $\mathrm{AdS}_{4}$ space. We exploit this to determine the nature of the equilibrium states realized at long times after the quench of this two dimensional type II superconductor in a perpendicular external uniform magnetic field $B_{0}$. This holographic superconductor exhibits the generic lower $\left(B_{c 1}(T)\right)$ and upper $\left(B_{c 2}(T)\right)$ critical fields. For $B_{0}<B_{c 1}(T)$ the magnetic field is completely expelled revealing the Meissner phase, while the superconductivity is destroyed when $B_{0}>B_{c 2}(T)$. Abrikosov lattices appear in the range $B_{c 1}(T)<B_{0}<B_{c 2}(T)$ that realize various configurations in the form of hexagonal, square and slightly irregular square lattices pending the magnetic field strength and the influence of finite size boundaries. We show this to be consistent with the expectations of Ginzburg-Landau theory where the upper and lower critical fields are associated with the inverse squares of the coherence length and magnetic penetration depth, respectively.
\end{abstract}

DOI: 10.1103/PhysRevD.105.L021901

\section{INTRODUCTION}

A well-known property of the type II superconductors is the quantization of the magnetic flux in the mixed state, where the magnetic field penetrates in the form of vortices that combine with the magnetic field into quantized fluxoids each carrying a quantized magnetic flux $\Phi_{0}=h c / 2 e$, forming an Abrikosov lattice [1]. The phenomenology is governed by the Ginzburg-Landau (GL) theory revolving around the parameter $\kappa=\lambda / \xi$, in terms of the magnetic penetration depth $\lambda$ and the coherence length $\xi$ of the order parameter that are pending microscopic conditions. In a conventional superconductor these can be computed by

\footnotetext{
*chuanyinxia@foxmail.com

thbzeng@yzu.edu.cn

*ytian@ucas.ac.cn

\$cmchen@phy.ncu.edu.tw

"jan@lorentz.leidenuniv.nl
}

Published by the American Physical Society under the terms of the Creative Commons Attribution 4.0 International license. Further distribution of this work must maintain attribution to the author(s) and the published article's title, journal citation, and DOI. Funded by SCOAP. employing the Bardeen-Cooper-Schrieffer (BCS) theory. When $\kappa<1 / \sqrt{2}$ the interaction between vortices is attractive resulting in a complete expulsion of the flux in the superconducting regions and a Meissner phase is realized. On the other hand, for $\kappa>1 / \sqrt{2}$, the vortices repel each other and the type II state characterized by the Abrikosov fluxoid lattice is formed in a magnetic field [2-6]. For reviews of the GL theory see for instance [7-12].

The applications of the AdS/CFT correspondence [13-15] or "holographic duality" to this condensed matter inspired context [16-18] jump started with the discovery $[19,20]$ that superconductivity can be described in a gravitational dual. One adds a charged scalar field to the Einstein-Maxwell theory describing the planar black hole living in the deep interior of the AdS bulk. Upon lowering temperature this scalar field may acquire a finite amplitude in the bulk. This translates in the boundary into a VEV without source signalling the spontaneous breaking of the $U(1)$ symmetry and the onset of superconductivity, as confirmed by the presence of the super current in the probe limit conductivity [20]. The magnetic field in the boundary is in turn dual to a magnetically charged black brane. However, the determination of the precise effects of magnetic fields on holographic superconductors has been 
proven to be a hazardous affair, which has not been settled up to now despite a number of attempts [21-23].

According to the probe limit the GL parameter $\kappa$ indicates that it is invariably of the type II kind [24-26]. Yet another approach is to involve the time evolution by attempting to solve the time independent equation of motions (EoMs) for the scalar and gauge fields in the bulk spacetime to look for equilibrium solutions [27-34]. One is confronted here with a highly nonlinear dynamical system where one got as of yet not beyond the description of the formation of a single vortex [27-30], while a static vortex lattice solution was only addressed relying on a perturbative method [31-34].

Here we will present a number of definitive answers in this regard. We accomplish the feat to solve the holographic equations describing the full time evolution within the limitations of the minimal holographic set up that we just discussed, in the physical setting of a quench involving the magnetic field. We depart from zero field, suddenly switching on a magnetic field to then track how the magnetic flux penetrates. A particular benefit of the holographic approach is the "first principle" treatment of dissipation. The system thermalizes and equilibrium sets in at long times. In a forthcoming publication we will discuss in more detail the transient dynamics that is similar to the outcomes of time dependent Ginzburg-Landau theory [35,36]. Here we will focus on what this has to tell about the equilibrium states in the presence of the field. Our results are summarized in the figures: the nature of the vortex lattice (Fig. 2), the temperature dependence of the coherence length and the penetration depth (Fig. 3), and the magnetization as function of field (Fig. 4). Last but not least, we determine the phase diagram at temperatures close to $\mathrm{T}_{c}$ (Fig. 5).

\section{HOLOGRAPHIC MODEL}

We depart from the standard set up for the minimal holographic superconductor, the usual Einstein bulk action with an Abelian-Higgs term describing the complex scalar added. In units of $\hbar=c=G_{N}=1$, the latter reads [19,20],

$$
S=\int d^{4} x \sqrt{-g}\left(-\frac{1}{4} F^{2}-|D \Psi|^{2}-m^{2}|\Psi|^{2}\right),
$$

where $F_{\mu \nu}=\partial_{\mu} A_{\nu}-\partial_{\nu} A_{\mu}$ and $D_{\mu}=\nabla_{\mu}-i q A_{\mu}$ with the charge of scalar field $q=2 e$ ("Cooper pair"). In the minimal setup one just takes the $\mathrm{AdS}_{4}$ black hole background, in Eddington-Finkelstein coordinates

$$
d s^{2}=\frac{\ell^{2}}{z^{2}}\left(-f(z) d t^{2}-2 d t d z+d x^{2}+d y^{2}\right),
$$

where $\ell$ is the AdS radius, $z$ is the radial coordinate of the AdS bulk and $f(z)=1-\left(z / z_{h}\right)^{3}: z=0$ is the AdS boundary while $z=z_{h}$ is the horizon. As is well established, ignoring completely the effects of finite density and the gravitational backreaction of the scalar field on the geometry limits the applicability of this set up to relatively high temperatures. At temperatures $\ll T_{c}$ the backreaction on the geometry becomes important but it is presently still too difficult to compute these in a dynamical, inhomogeneous setting.

The dual field theory lives at $z=0$, and the information needed for the dual superconductor can be read off from the asymptotics of the bulk fields approaching the boundary. These can be obtained by solving the dynamic coupled equations of motion for $\Psi$ and $A_{\mu}$,

$$
\left(D^{2}-m^{2}\right) \Psi=0, \quad \nabla_{\mu} F^{\mu \nu}=i \Psi^{*} D^{\nu} \Psi-i \Psi\left(D^{\nu} \Psi\right)^{*} .
$$

The spontaneous breaking of the local $U(1)$ symmetry in the field theory is encoded by the induced nonzero expectation value $\Psi^{(2)}$ of the scalar operator dual to $\Psi$ in the bulk, which follows from the asymptotic behavior of $\Psi$ near the boundary,

$$
\Psi(z \sim 0, t, x, y) \approx \Psi^{(1)}(t, x, y) z+\Psi^{(2)}(t, x, y) z^{2},
$$

where the source $\Psi^{(1)}$ is set to be zero as a boundary condition when solving the model. Furthermore, in order to introduce a magnetic field in the dual holographic superconductor, the gauge fields on the boundary should be dynamic. In the gauge $A_{z}=0$, the behavior of the gauge fields on the boundary corresponds with,

$$
A_{\mu}(z \sim 0, t, x, y) \approx a_{\mu}(t, x, y)+b_{\mu}(t, x, y) z
$$

where $a_{\mu}$ is identified with the gauge field of the boundary theory, while $b_{\mu}$ is according to the holographic dictionary related to the current $j_{\mu}$ as $j_{\mu}=-b_{\mu}-\partial_{\mu} a_{t}+\partial_{t} a_{\mu}$ in Eddington coordinates. We control the charge density by $\rho=-b_{t}$ which is equivalent to tuning the temperature. In the superconductor regime we fix $j_{x}=j_{y}=0$ as the Neumann boundary condition for $A_{x}$ and $A_{y}$ at $z=0$. This is different from the neutral superfluid that is captured by Dirichlet boundary conditions $a_{x}=a_{y}=0$, leading to vortex lattice solutions studied by [37-42].

We create a finite size "square" of superconducting matter surrounded by a metal by switching of both the scalar-and gauge fields outside this square. Similar to experimental flux penetration circumstances we then implement the following protocol. We prepare a homogeneous superconducting state at a fixed temperature as the initial configuration by the Newton-Raphson method. We then apply suddenly an uniform external magnetic field at $t=0$ by turning on $A_{x}(t=0, z, x, y)=-B_{0} y / 2$ and $A_{y}(t=0, z, x, y)=B_{0} x / 2$ and track in time how the magnetic flux penetrates into the superconducting square. We solve this dynamical problem by combining a RungeKutta method in the time direction and a Chebyshev 
spectral method for the other three coordinates $z, x, y$, similar to previous work on vortex lattice formation in a rotating holographic superfluid [38]. For the numerical simulations we choose as parameters $\ell=1, m^{2}=-2$ and $q=1$ (implying $\Phi_{0}=2 \pi$ ).

\section{ABRIKOSOV LATTICES}

A typical outcome is shown at a temperature close to $T_{c}$ in Fig. 1 at long times where the system has completely equilibrated. These correspond with a typical hexagonal Abrikosov lattice (panels a, b), while the internal structure of the fluxoid is according to expectations (panel c). The widths of the flux lines $\lambda$ and the order parameter defects $\xi$ can be fitted from the profile of the magnetic field and the expectation value of the order parameter using $B(r) \sim$ $0.3949 \exp (-r / \lambda)$ and $\langle O(r)\rangle \sim 1.3693 \tanh (r / \sqrt{2} \xi)$, respectively. For $T=0.95 T_{c}$ and $B_{0}=0.428$ we find in this way that $\lambda \sim 1.579$ and $\xi \sim 1.1$. This corresponds with the GL parameter $\kappa \sim 1.435$, consistent with type II behavior.

According to the GL theory, lattices with equilateral triangles are at a slightly lower free energy than square lattices. This rests in essence on the simple wisdom that in two dimensions a closed packed lattice just corresponds with the triangular one, where every vortex is surrounded by a hexagonal array of other vortices. In this array, the nearest neighbor distance can be evaluated from the averaged value of the magnetic field in a vortex $\langle B\rangle$ to be,

$$
a_{\triangle}=\left(\frac{4}{3}\right)^{\frac{1}{4}} a_{\square} \approx\left(\frac{4}{3}\right)^{\frac{1}{4}}\left(\frac{\Phi_{0}}{\langle B\rangle}\right)^{\frac{1}{2}} .
$$

Thus, for a given flux density, $a_{\square}<a_{\triangle}$. Taking into account the mutual repulsion of the vortices, it is reasonable

(a)
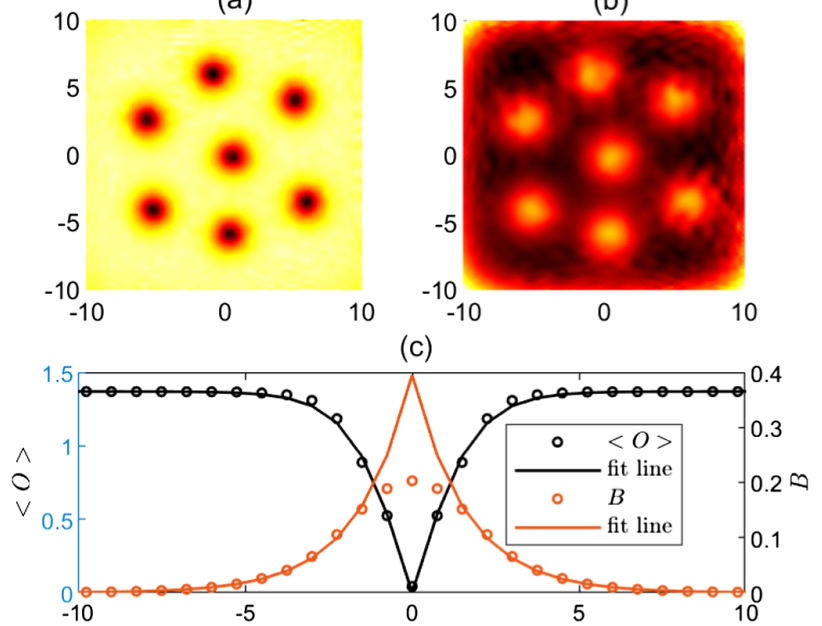

FIG. 1. A hexagonal lattice at $T=0.95 T_{c}, B_{0}=0.428$ : configurations of (a) the order parameter, (b) the magnetic field, and (c) the radial profiles of the order parameter and magnetic field in a single vortex. to expect that the structure with the largest separation to the nearest neighbors will be favored. From Eq. (6) the distance between two nearest vortices can be computed to be $a_{\triangle} \approx 1.075 \sqrt{2 \pi / 0.178} \approx 6.39$, close to the numerical simulation result $a_{\triangle} \approx 6.63$.

However, different things are happening according to our simulations when the vortex density is increased by ramping up the magnetic field. Two typical examples are shown in Fig. 2. For the case with $T=0.9 T_{c}, B_{0}=0.8$, we find 9 vortices now forming a square pattern. In this case, the distance between vortices is $a_{\square} \approx 5.02$ (close to the value from (6) $a_{\square} \approx 5$.05); this is not sufficiently small while the finite size of our square patch with its straight boundaries prohibits the formation of a hexagon pattern. For the case $T=0.82 T_{c}, B_{0}=1.7$, the vortex number is 30 , while the distance between two nearest vortices computed by (6) $a_{\triangle} \approx 3.43$ is now close to the numerical simulation value $a_{\triangle} \approx 3.214$. The finite size effect is now moderated, and the hexagonal pattern is favored again. However, the array does not admit a perfect lattice configuration, which is again explained by finite size effects-a substantial number of the vortices are still close to the boundary.

In summary, our holographic simulations are consistent with the expectation. We find that the lattice configurations depend on (a) the density of vortices as controlled by the magnetic field, (b) the spatial extent of the normal core of the fluxoids controlled by temperature, and (c) the finite size effects associated with the boundary. When (c) is dominating, a square lattice configuration is preferred when the vortex number is small and close to the square of an integer. Otherwise large number of vortices always forms
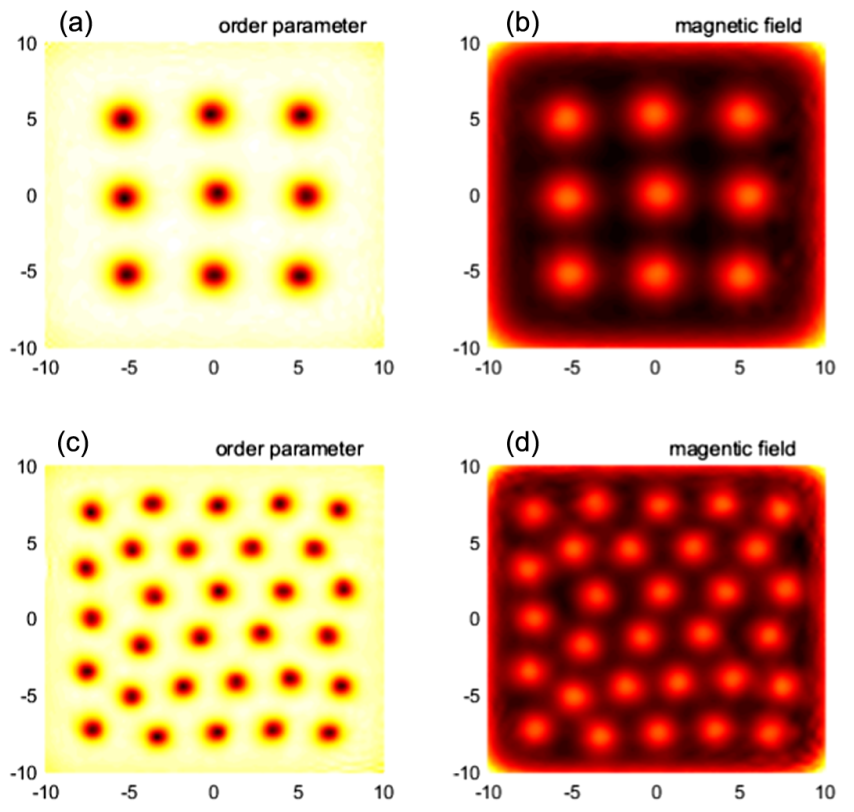

FIG. 2. Two representative vortex lattice solutions: (a-b) $T=0.9 T_{c}, B_{0}=0.8$, and (c-d) $T=0.82 T_{c}, B_{0}=1.7$. 


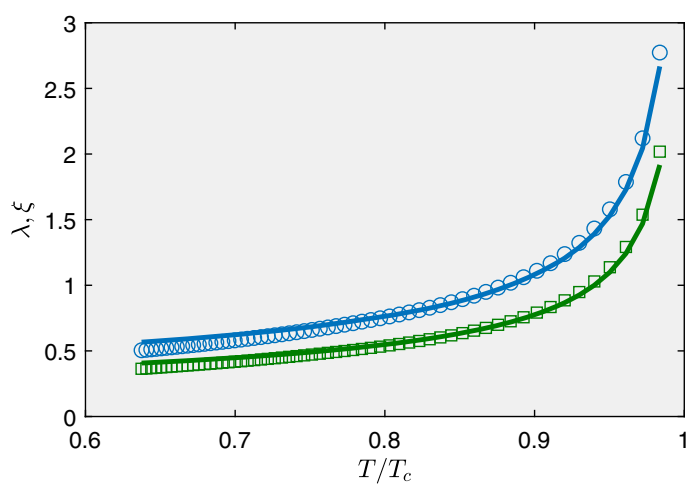

FIG. 3. The temperature dependence of $\xi$ (green) and $\lambda$ (blue) according to the numerics, fitted by the GL formulas Eq.'s (7) using as free parameters $\xi_{0}=0.3313$ and $\lambda_{0}=0.4825$.

distorted hexagonal configurations, as also observed experimentally in mesoscopic superconducting islands (superconducting PB [43], cuprate thin films [44].)

Finally, by increasing the magnetic field until it approaches the upper critical fields $B_{c 2}$, we find that the superconducting area inside the square shrinks, leaving behind a superconducting island with vortices crowded together without a well defined nearest vortex distance.

The size of the normal core of the fluxoid is determined by the coherence length $\xi$ and according to GL theory [11] for $T$ close to $T_{c}$ this length and the penetration depth $\lambda$ determining the spatial extend of the magnetic flux should vary according to,

$\xi \sim 0.74 \xi_{0}\left(1-T / T_{c}\right)^{-1 / 2}, \quad \lambda \sim \frac{\lambda_{0}}{\sqrt{2}}\left(1-T / T_{c}\right)^{-1 / 2}$,

Our results shown in Fig. 3 closely comply to these expectations. Both $\xi$ and $\lambda$ are almost independent of $B_{0}$ and the temperature dependence follows closely these formulas; for example we find a deviation of at most $\sim 3 \%$ at $T=0.95 T_{c}$. This reveals that $\xi_{0} \sim 0.3313$ and $\lambda_{0} \sim$ 0.4825 implying the GL parameter to be $\kappa \sim 1.3916$ for this holographic superconductor. These formula's are in general expected to be only obeyed close to $T_{c}$ but we find that it applies quite well down to temperatures as small as $\sim 0.6 T_{c}$ (Fig. 3). Notice that in this regard it is quite similar as to conventional BCS superconductors where it is well established that these GL relations work well down to the lowest temperatures (e.g., niobium [45]).

\section{MAGNETIZATION}

The magnetization $M$ is the thermodynamical quantity of choice to distinguish the Meissner- and vortex lattice phase. This is defined as,

$$
M\left(B_{0}\right)=B_{0}-\langle B(x, y)\rangle
$$

where $B_{0}$ is the value of the applied external magnetic field, while $B(x, y)$ is the magnetic field distribution in the

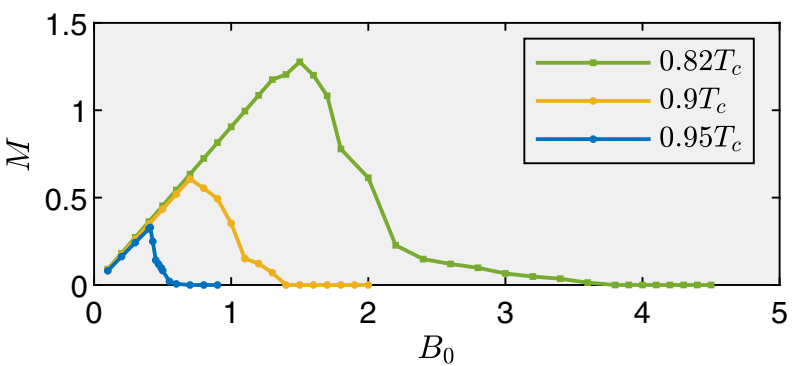

FIG. 4. The magnetization $M$ defined in Eq. (8) for three different temperatures $T=0.82 T_{c}, 0.9 T_{c}$ and $0.95 T_{c}$, from the curves we can read the two critical fields where the magnetization reaches its maximal value and reduces to zero respectively.

equilibrium state. When the added external field increases from zero to $B_{c 1}$, the magnetic field is completely expelled and there is no magnetic field inside the sample, $M=B_{0}$. On the other hand, above $B_{c 2}$, the superconductivity is completely destroyed, $B(x, y)=B_{0}$ and $M$ is zero. In the interval $B_{c 1}<B_{0}<B_{c 2}$ the mixed state is stable with the ramification that the magnetization $M$ will decrease from $B_{c 1}$ to zero gradually. In Fig. 4 we show the magnetization versus $B_{0}$ for three different temperatures confirming these expectations.

\section{PHASE DIAGRAM}

From these magnetization curves one can directly read off the values of two critical magnetic fields. $B_{c 1}$ corresponds with the field where the linear increase of $M$ terminates, while $B_{c 2}$ is the field value where $M$ vanishes. The outcomes are summarized in our final result in the form of the phase diagram as function of temperature and field, Fig. 5. The critical fields actually conform closely to the predictions of the canonical GL analysis [10,11] that typically applies in conventional BCS superconductors,

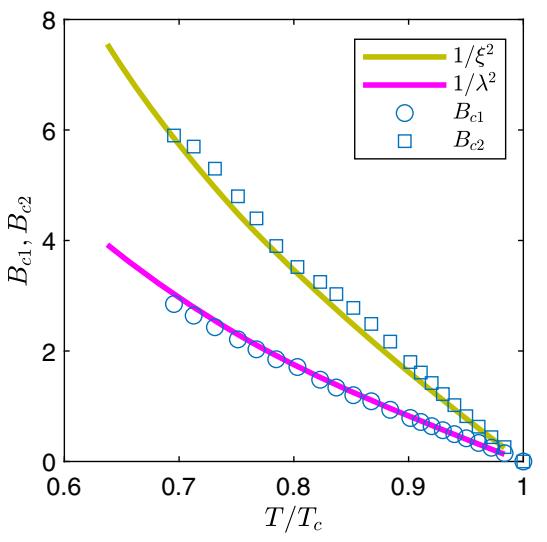

FIG. 5. The phase diagram of holographic superconductor in an external magnetic field. The circles and squares indicate the lower- and upper critical fields as determined from the numerical results for the magnetization (Fig. 4) while the lines follow from the expectations of GL theory, Eqs. (10), (11). 
see, e.g., $[45,46]$. Here $B_{c 2}$ is estimated by the instance where the separation of the fluxoids becomes of order of the core size, where no longer a superconducting path remains for the transport current. It follows from this criterium that,

$$
B_{c 2}=\frac{\Phi_{0}}{2 \pi \xi^{2}}=\xi^{-2},
$$

where $\pi \xi^{2}$ is the size of the Abrikosov unit cell. Using the result (7),

$$
B_{c 2} \approx 16.64\left(1-T / T_{c}\right),
$$

The lower critical magnetic field $B_{c 1}$ is determined by the instance where the first vortex is created,

$$
B_{c 1} \approx \frac{\Phi_{0}}{2 \pi \lambda^{2}}=\lambda^{-2},
$$

where $\pi \lambda^{2}$ corresponds with the area where the magnetic field penetrates. These simple relations fit the numerically determined results for the critical fields very well, see Fig. 5.

\section{SUMMARY}

The quantitative description of the formation of the Abrikosov lattice in conventional type II superconductors is a classic success of Ginzburg-Landau theory resting on the microscopic BCS theory. Using dynamical quenches to obtain an unbiased view on the equilibrium states we have charted the outcomes for the minimal holographic superconductor. We find an excellent agreement with the expectations of the GL theory also at temperatures quite some distance away from the zero field critical temperature. This emphasizes that in phenomenological regards holographic superconductors behave in a way that is strikingly similar to the BCS variety. This is hindsight not surprising - although the metallic states described by holography are very different from the simple Fermi gas underlying BCS the description of the superconducting state is characterized by striking similarities (see, e.g., Ref. [18]).

This study is limited to the "minimal" set up that is expected to be representative for generic holographic superconductors at elevated temperatures near $T_{c}$. There is still quite some terrain to explore in the low temperature realms where finite density and especially the gravitational backreaction become important, forming still quite a computational challenge. Similarly, it would be of interest to extend this to three space dimensions. Yet another great potential for holography is in the description of vortex dynamics. Here we have been focussed on the equilibrium that sets in a long time after the quench. However, there appears to be much to be learned regarding the way that flux penetrates the superconductor, while there is a myriad of other nonequilibrium circumstances that can be studied using time dependent holography. These will be the subject of forthcoming publications.

\section{ACKNOWLEDGMENTS}

This work is supported by the National Natural Science Foundation of China under Grant No. 11675140 (CYX, HBZ), No. 11975235, No. 12035016 (YT), and the Ministry of Science and Technology of the R. O. C. under the Grants No. MOST 109-2112-M-008-010, No. 1102112-M-008-009 (CMC).
[1] A. A. Abrikosov, Sov. Phys. JETP 5, 1174 (1957).

[2] E. H. Brandt, Rep. Prog. Phys. 58, 1465 (1995).

[3] E. B. Bogomolny, Sov. J. Nucl. Phys. 24, 449 (1976).

[4] E. B. Bogomolny and A. I. Vainshtein, Sov. J. Nucl. Phys. 23, 588 (1976).

[5] L. Jacobs and C. Rebbi, Phys. Rev. B 19, 4486 (1979).

[6] A. J. Beekman and J. Zaanen, Front. Phys. (Beijing) 6, 357 (2011).

[7] V. L. Ginzburg and L. D. Landau, Zh. Eksp. Teor. Fiz. 20, 1064 (1950).

[8] P. G. de Gennes, Superconductivity of Metals and Alloys (Benjamin, New York, 1966).

[9] D. Saint-James, G. Sarma, and E. J. Thomas, Type-II Superconductivity (Benjamin, New York, 1969).

[10] G. Blatter, M. V. Feigel'man, V. B. Geshkenbein, A. I. Larkin, and V. M. Vinokur, Rev. Mod. Phys. 66, 1125 (1994).
[11] M. Tinkham, Introduction to Superconductivity (Dover Publication, Mineola, New York, 2004).

[12] B. Rosenstein and D. Li, Rev. Mod. Phys. 82, 109 (2010).

[13] J. M. Maldacena, Adv. Theor. Math. Phys. 2, 231 (1998).

[14] S. S. Gubser, I. R. Klebanov, and A. M. Polyakov, Phys. Lett. B 428, 105 (1998).

[15] E. Witten, Adv. Theor. Math. Phys. 2, 253 (1998).

[16] J. Zaanen, Y.-W. Sun, Y. Liu, and K. Schalm, Holographic Duality in Condensed Matter Physics (Cambridge University Press, Cambridge, England, 2015).

[17] S. A. Hartnoll, A. Lucas, and S. Sachdev, Holographic Quantum Matter (MIT Press, Cambridge, MA, 2018).

[18] J. Zaanen, arXiv:2110.00961.

[19] S. S. Gubser, Phys. Rev. D 78, 065034 (2008).

[20] S. A. Hartnoll, C. P. Herzog, and G. T. Horowitz, Phys. Rev. Lett. 101, 031601 (2008).

[21] E. Nakano and W. Y. Wen, Phys. Rev. D 78, 046004 (2008). 
[22] T. Albash and C. V. Johnson, J. High Energy Phys. 09 (2008) 121.

[23] T. Albash and C. V. Johnson, arXiv:0906.0519.

[24] O. C. Umeh, J. High Energy Phys. 08 (2009) 062.

[25] S. A. Hartnoll, C. P. Herzog, and G. T. Horowitz, J. High Energy Phys. 12 (2008) 015.

[26] H. B. Zeng, C. Y. Xia, and H. Q. Zhang, J. High Energy Phys. 03 (2021) 136.

[27] M. Montull, A. Pomarol, and P. J. Silva, Phys. Rev. Lett. 103, 091601 (2009).

[28] O. Domenech, M. Montull, A. Pomarol, A. Salvio, and P. J. Silva, J. High Energy Phys. 08 (2010) 033.

[29] V. Keranen, E. Keski-Vakkuri, S. Nowling, and K. P. Yogendran, Phys. Rev. D 81, 126012 (2010).

[30] T. Albash and C. V. Johnson, Phys. Rev. D 80, 126009 (2009).

[31] K. Maeda, M. Natsuume, and T. Okamura, Phys. Rev. D 81, 026002 (2010).

[32] Y. Y. Bu, J. Erdmenger, J. P. Shock, and M. Strydom, J. High Energy Phys. 03 (2013) 165.

[33] A. Donos, J. P. Gauntlett, and C. Pantelidou, J. High Energy Phys. 07 (2020) 095.

[34] G. Tallarita and R. Auzzi, J. High Energy Phys. 01 (2020) 056.
[35] A. T. Dorsey, Phys. Rev. B 46, 8376 (1992).

[36] Q. Du, M.. D. Gunzburger, and J. S. Peterson, Phys. Rev. B 46, 9027 (1992).

[37] Ó. J. C. Dias, G. T. Horowitz, N. Iqbal, and J. E. Santos, J. High Energy Phys. 04 (2014) 096.

[38] C. Y. Xia, H. B. Zeng, H. Q. Zhang, Z. Y. Nie, Y. Tian, and X. Li, Phys. Rev. D 100, 061901(R) (2019).

[39] X. Li, Y. Tian, and H. Zhang, J. High Energy Phys. 02 (2020) 104.

[40] P. Wittmer, C. M. Schmied, T. Gasenzer, and C. Ewerz, Phys. Rev. Lett. 127, 101601 (2021).

[41] C. Ewerz, A. Samberg, and P. Wittmer, J. High Energy Phys. 11 (2021) 199.

[42] A. Srivastav and S. Gangopadhyay, arXiv:2105.15045.

[43] U. Essmann and H. Träuble, Phys. Lett. 24A, 526 (1967).

[44] F. S. Wells, A. V. Pan, X.-R. Wang, S. A. Fedoseev, and H. Hilgenkamp, Sci. Rep. 5, 8677 (2015).

[45] E. Navarro, C. Monton, J. Pereiro, A. C. Basaran, and I. K. Schuller, Phys. Rev. B 92, 144512 (2015).

[46] I. Zaytseva, A. Abaloszew, B. C. Camargo, Y. Syryanyy, and M. Z. Cieplak, Sci. Rep. 10, 19062 (2020). 